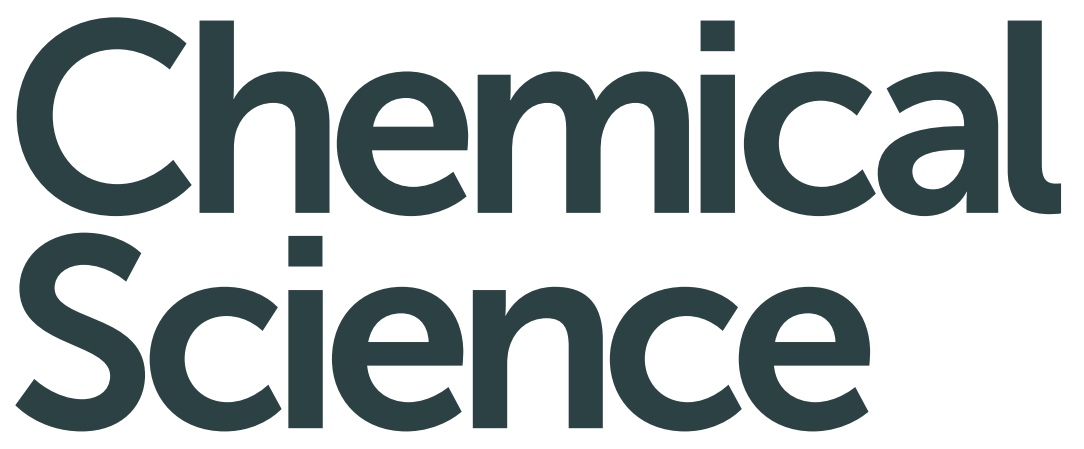

rsc.li/chemical-science

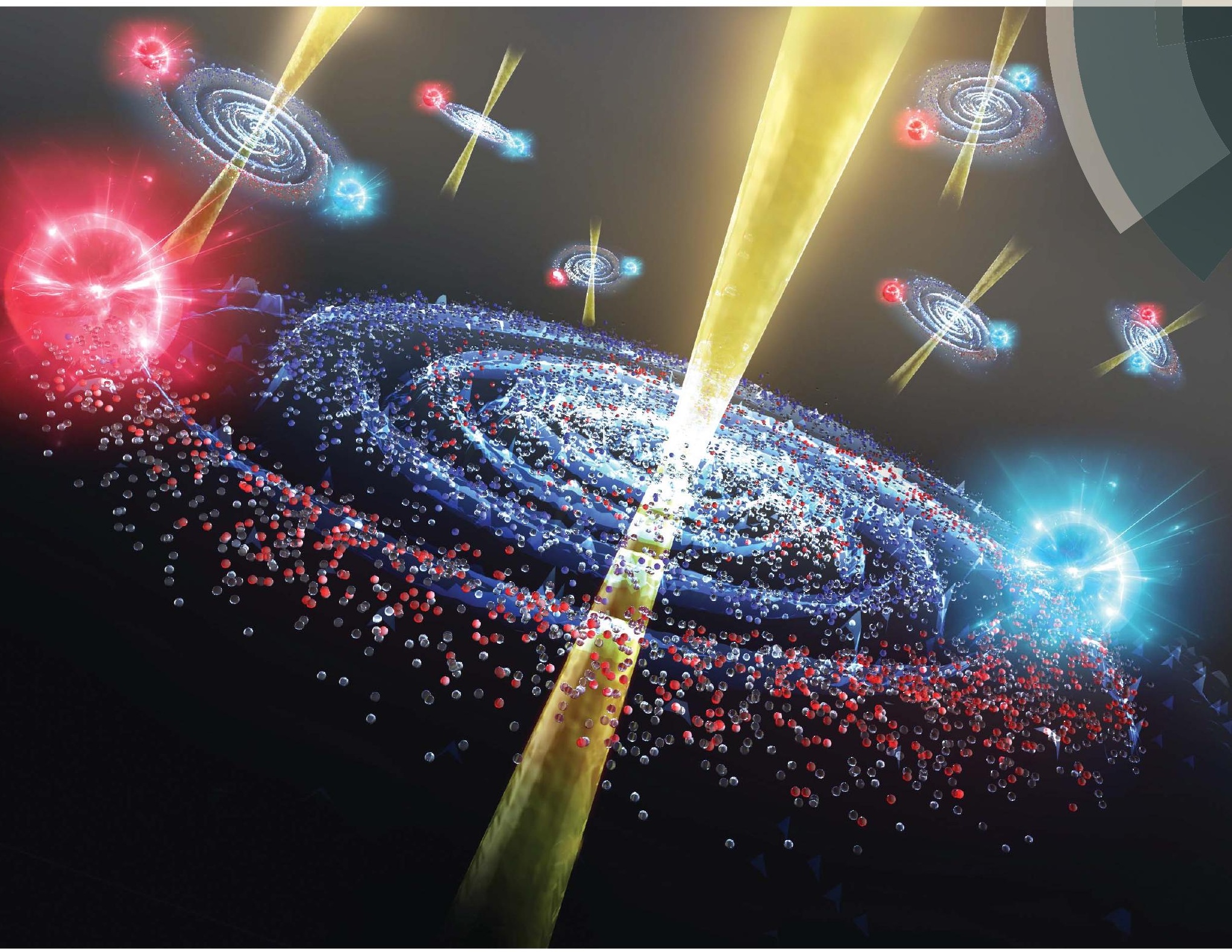

ISSN 2041-6539 
Check for updates

Cite this: Chem. Sci., 2019, 10, 9203

๑ All publication charges for this article have been paid for by the Royal Society of Chemistry

\title{
Exciplex emissions derived from exceptionally long-distance donor and acceptor molecules $\uparrow$
}

\author{
Yong-Jin Pu, (D)*ab Yuki Koyama, ab Daisuke Otsuki, ${ }^{\text {b }}$ Minjun Kim, ${ }^{\text {a Hiroya Chubachi, }}{ }^{\text {b }}$ \\ Yuki Seino, ${ }^{a}$ Kazushi Enomoto ${ }^{a}$ and Naoya Aizawa (D) ac
}

Intermolecular electron-hole coupling in organic semiconductor excited states plays important roles in organic light-emitting diodes and organic photovoltaics, and the distance of the coupling is typically only on the order of a few nanometers. Here, we report exceptionally long-distance coupled exciplex emissions between electron-donor and electron-acceptor molecules even with a $70 \mathrm{~nm}$-thick spacer layer. Donor/spacer ( 70 nm)/acceptor-type stacked films showed a low-energy band emission, which is not ascribed to the emission of the donor, spacer, and acceptor themselves, but well corresponds to the energy difference between the highest occupied molecular orbital of the donor and the lowest unoccupied molecular orbital of the acceptor. Delayed transient photoluminescence (PL) and electroluminescence (EL) decays and PL quenching by oxygen at the low-energy band were observed and are consistent with the characteristics of the exciplex species.

Received 26th August 2019

Accepted 9th September 2019

DOI: $10.1039 / \mathrm{c} 9 \mathrm{sc} 04262 \mathrm{~h}$

rsc.li/chemical-science

host for phosphorescent and fluorescent emitters in OLEDs

\section{Introduction}

An exciplex is an intermolecular complex in an excited state, formed from electron-donor and electron-acceptor molecules. These have been widely studied especially in the context of photosynthesis chemistry, in an effort to understand photoinduced electron transfer and to create long-lived charge-separated states with donor-spacer-acceptor system frameworks, including electron-donating porphyrin derivatives, electronaccepting fullerenes, carbon nanotubes, and $\pi$-conjugated molecules. ${ }^{1-3}$ The energy level of an exciplex state is lower than that of each independent electron-donor and electron-acceptor molecule, and thus its emission wavelength becomes bathochromically-shifted from the original emission of each molecule. Photoluminescence (PL) quantum yields of exciplex emissions tend to be low, and therefore the exciplex emissions have not been widely utilized in organic light-emitting diode (OLED) applications. ${ }^{4,5}$ However, Goushi et al. recently used the exciplex state between a hole-transporting layer (HTL) and an electron-transporting layer (ETL), in which the energy difference between the excited singlet state and triplet state becomes small and favourable to achieve thermally activated delayed fluorescence (TADF), enhancing the internal quantum efficiency of fluorescent OLEDs. ${ }^{6}$ Park and Sun $e t$ al. reported an exciplex co-

${ }^{a}$ RIKEN Center for Emergent Matter Science (CEMS), Wako, Saitama 351-0198, Japan. E-mail: yongjin.pu@riken.jp

${ }^{b}$ Graduate School of Organic Materials Science, Yamagata University, Yonezawa, Yamagata 992-8510, Japan

'JST-PRESTO, Kawaguchi, Saitama 332-0012, Japan

$\dagger$ Electronic supplementary information (ESI) available. See DOI: $10.1039 / \mathrm{c} 9 \mathrm{sc} 04262 \mathrm{~h}$ having a high external quantum efficiency (EQE) of approximately $30 \% .^{7,8}$ An exciplex can be considered as a partially charge-transfer (CT) excited state, and it is also important to understand and enhance charge separation of bound holeelectron pairs at the $\mathrm{p}-\mathrm{n}$ interface for organic photovoltaics. ${ }^{9,10}$ In organic molecules with low dielectric constants in particular, hole-electron pairs are tightly bound within small exciton Bohr radii.

For exciplex formation, the distance between electron-donor and electron-acceptor molecules has to be very small, on the order of a few nanometers at most. ${ }^{11-13}$ However, Nakanotani et al. observed exciplex emissions derived from long-distance coupled donor and acceptor molecules with a $10 \mathrm{~nm}$ thick spacer layer, ${ }^{14}$ and then, Chapran et al., Li et al., and Sych et al. also observed similar exciplex emissions with a several nanometer spacer layer, using different types of donor and acceptor molecules. ${ }^{15-17}$ Colella et al. reported that donor and acceptor molecules diluted in a host matrix (i.e. a spacer molecule) showed efficient exciplex emission..$^{18}$ Supporting those reports, Deotare et al. found that bound electron-hole pairs moved to a distance of approximately $10 \mathrm{~nm}$ at the p-n interface. ${ }^{19}$ These extraordinarily long-distance electron-hole couplings between the donor and acceptor molecules are still not well understood, and developing and understanding such exotic exciplex states (i.e. long-distance coupled excited CT states) are both a fascinating and challenging direction of study.

Here we report abnormally long-distance exciplex emissions in OLEDs even with an $\sim 70 \mathrm{~nm}$-thick spacer layer between electron-donor molecules and electron-acceptor molecules. Various types of devices and stacked films were fabricated and 
investigated to prove that the emergent low-energy band emission originates from the long-distance coupled exciplex species and to exclude other possibilities. Electroluminescence (EL) and transient EL of the devices with different spacer layers were measured to compare with PL and transient PL of the donor:acceptor mixed or stacked films. A device exclusively showing electromer emission was fabricated to judge the relevance of the electromer to the low-energy band emission. Various donor molecules in the OLEDs were systematically used, while the types of spacer and acceptor molecules were fixed, to investigate the influence of the energy difference between the donor and acceptor molecules on the low-energy band emission. Oxygen dependence and temperature dependence of the PL were measured to obtain more confident evidence that the excited triplet state is involved in the low-energy band emission as a TADF-type exciplex. The type of spacer molecule was also changed to help understand the role of the interfaces between the donor and the spacer and between the spacer and the acceptor in the emission mechanism.

\section{Results and discussion}

1,1'-Bis(di-4-tolyl-aminophenyl)cyclohexane (TAPC), 9,10bis(3,5-dimethoxyphenyl)anthracene (DMA), and 9,10-bis(3,5dicyanophenyl)anthracene (DCA) were used as the donor molecule (D), spacer molecule (S), and acceptor molecule (A), respectively. Chemical structures of the compounds, PL spectra of their neat films and co-evaporated mixed films, and transient PL decays are shown in Fig. 1 and PL quantum yields of the films are summarized in Table $\mathrm{S} 1 . \dagger$ Co-evaporated films with each combination of TAPC:DMA (D:S), DMA:DCA (S:A), and TAPC:DCA (D:A) showed bathochromically shifted PL compared to the PL of their respective neat films (D, S, and A), and these red-shifted emissions are ascribed to exciplex emissions from the D:S, S:A, and D:A complexes. PL decays of the D:S, S:A, and $\mathrm{D}$ :A complexes were on the submicrosecond scale (125-299 ns), although the PL decays from the neat films of D, S, and A themselves were on the subnanosecond scale (0.4-0.98 ns), supporting the exciplex formation of D:S, S:A, and D:A (Tables $\mathrm{S} 2$ and $\mathrm{S} 3 \dagger)$.

OLEDs with a structure of ITO/poly(3,4-ethylenedioxythiophene):poly(4-styrenesulfonate) (PEDOT:PSS) (30 $\mathrm{nm}) /$ TAPC (20 nm)/DMA (0, 10, 20, 30, 40, 50, 60, $70 \mathrm{~nm}) / \mathrm{DCA}$ (10 nm)/1,3-bis[3,5-di(pyridin-3-yl)phenyl]benzene ${ }^{20}$ (В3РyPB) $(40 \mathrm{~nm}) / \mathrm{LiF}(1 \mathrm{~nm}) / \mathrm{Al}$ were fabricated for this study. B3PyPB was used as an ETL and its chemical structure is shown in Fig. S1. $\dagger$ The energy diagram, EL spectra, and transient EL decay of the device are shown in Fig. 2. EQEs of the devices are summarized in Table S4. $\dagger$ The device without the spacer DMA (i.e. $0 \mathrm{~nm}$ spacer) showed two EL emissions, in which the high-energy band emission with a $\lambda_{\max }$ of $445 \mathrm{~nm}$ was identical to the PL spectrum of the acceptor DCA itself and the low-energy band emission with a $\lambda_{\max }$ of $550 \mathrm{~nm}$ was similar to the PL spectrum of the exciplex between the donor TAPC and the acceptor DCA. a<smiles>Cc1ccc(N(c2ccc(C)cc2)c2ccc(C3(c4ccc(N(c5ccc(C)cc5)c5ccc(C)cc5)cc4)CCCCC3)cc2)cc1</smiles><smiles>COc1cc(OC)cc(-c2c3ccccc3c(-c3cc(OC)cc(OC)c3)c3ccccc23)c1</smiles>

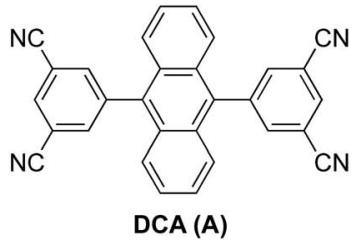

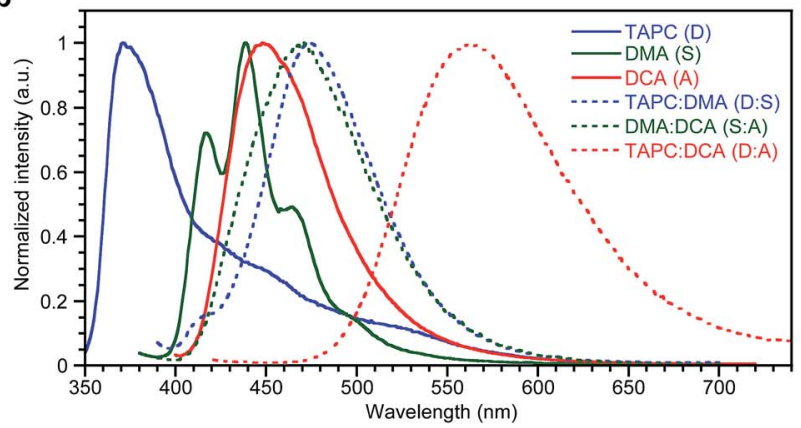

c

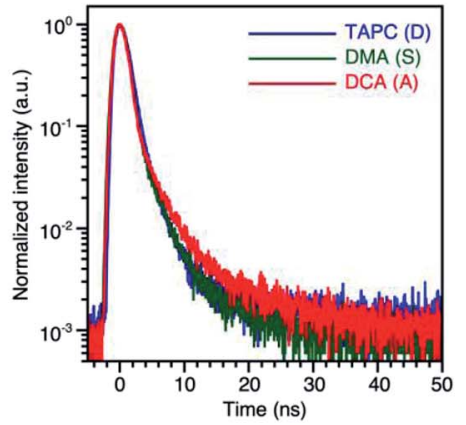

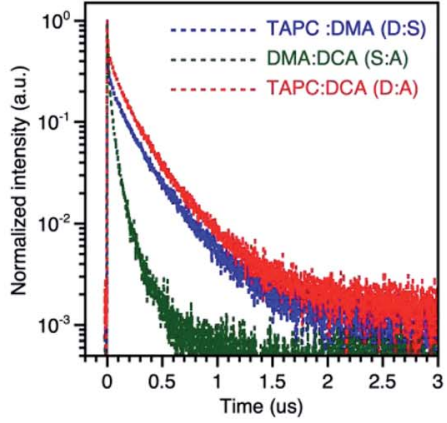

Fig. $1 \mathrm{PL}$ properties of the donor TAPC, the spacer DMA, and the acceptor DCA; (a) chemical structures of the compounds. (b) PL spectra of the neat films and the co-evaporated (1/1 wt/wt) films. TAPC $\left(\lambda_{\text {ex }} 330 \mathrm{~nm}\right), \mathrm{DMA}\left(\lambda_{\mathrm{ex}} 360 \mathrm{~nm}\right), \mathrm{DCA}\left(\lambda_{\mathrm{ex}} 380 \mathrm{~nm}\right)$, TAPC:DMA $\left(\lambda_{\mathrm{ex}} 370 \mathrm{~nm}\right), \mathrm{DMA}: \mathrm{DCA}$ $\left(\lambda_{\text {ex }} 370 \mathrm{~nm}\right)$, and TAPC:DCA $\left(\lambda_{\text {ex }} 400 \mathrm{~nm}\right)$. (c) Transient PL decay of the neat films and the co-evaporated $(1 / 1 \mathrm{wt} / \mathrm{wt})$ films: TAPC $\left(\lambda_{\mathrm{ex}} 280 \mathrm{~nm}\right.$, $\lambda_{\mathrm{em}}$ $380 \mathrm{~nm}), \mathrm{DMA}\left(\lambda_{\mathrm{ex}} 370 \mathrm{~nm}, \lambda_{\mathrm{em}} 440 \mathrm{~nm}\right), \mathrm{DCA}\left(\lambda_{\mathrm{ex}} 370 \mathrm{~nm}, \lambda_{\mathrm{em}} 470 \mathrm{~nm}\right)$, TAPC:DMA $\left(\lambda_{\mathrm{ex}} 370 \mathrm{~nm}, \lambda_{\mathrm{em}} 475 \mathrm{~nm}\right), \mathrm{DMA}: \mathrm{DCA}\left(\lambda_{\mathrm{ex}} 370 \mathrm{~nm}, \lambda_{\mathrm{em}} 470\right.$ $\mathrm{nm})$, and TAPC:DCA ( $\left.\lambda_{\mathrm{ex}} 370 \mathrm{~nm}, \lambda_{\mathrm{em}} 550 \mathrm{~nm}\right)$. 
a

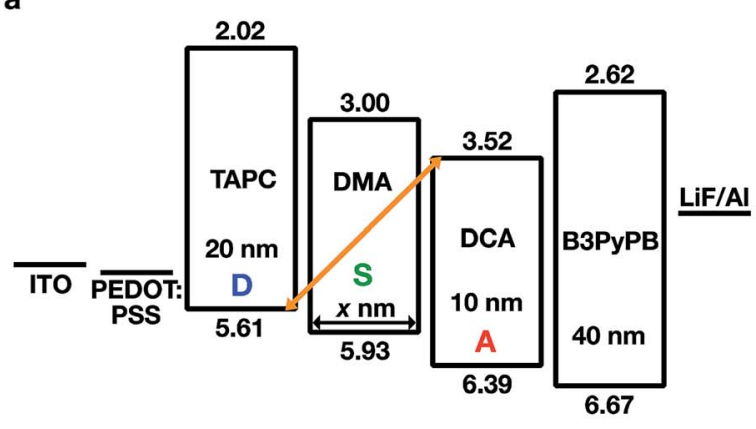

b

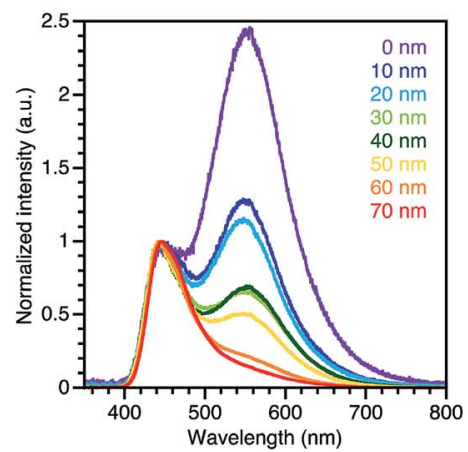

c

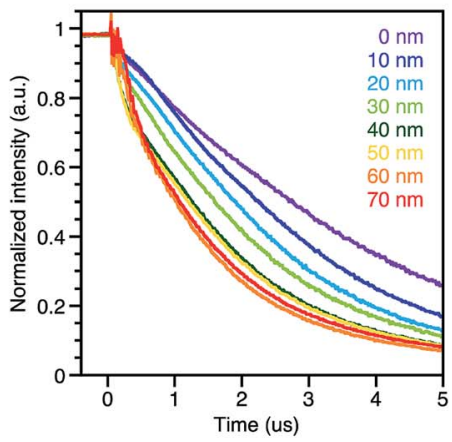

Fig. 2 Spacer-thickness dependent EL in the devices with the DMA spacer; (a) device structure. (b) $\mathrm{EL} \mathrm{spectra} \mathrm{at} 25 \mathrm{~mA} \mathrm{~cm}^{-2}$ with different spacer thicknesses from $0 \mathrm{~nm}$ to $70 \mathrm{~nm}$. (c) Transient EL decay at $50 \mathrm{~mA} \mathrm{~cm}{ }^{-2}$ with different spacer thicknesses from $0 \mathrm{~nm}$ to $70 \mathrm{~nm}$. The applied voltages are $5.50 \mathrm{~V}$ for $0 \mathrm{~nm}, 5.66 \mathrm{~V}$ for $10 \mathrm{~nm}, 6.41 \mathrm{~V}$ for $20 \mathrm{~nm}, 6.90 \mathrm{~V}$ for $30 \mathrm{~nm}, 7.60 \mathrm{~V}$ for $40 \mathrm{~nm}, 8.05 \mathrm{~V}$ for $50 \mathrm{~nm}, 8.58 \mathrm{~V}$ for $60 \mathrm{~nm}$, and $9.14 \mathrm{~V}$ for $70 \mathrm{~nm}$.

The low-energy band emissions became smaller with increasing the spacer layer thickness, but surprisingly the emission did not totally disappear even with a $70 \mathrm{~nm}$ thick spacer. Transient EL decay was also measured. The decay lifetime was long and on the microsecond scale, demonstrating that residual excitons in the device originate from long lifetime species such as exciplexes. The microsecond-scale transient EL component became smaller with increasing the spacer layer thickness, but this delayed component did not totally disappear even with abnormally thick spacers ( $\sim 70 \mathrm{~nm}$ thickness) between the donor and the acceptor layers. To exclude any possibility that the acceptor DCA or the donor TAPC is not involved in the low-energy band emission, the device $\mathrm{D} / \mathrm{S}$ without the acceptor DCA layer and the device S/A without the donor TAPC layer were fabricated, respectively, and their EL spectra are compared (Fig. S2 $\dagger$ ). The device D/S and the device S/A clearly did not show the low-energy band emission corresponding to those of the $\mathrm{D} / \mathrm{A}$ and $\mathrm{D} / \mathrm{S} / \mathrm{A}$ devices. The transient EL of the D/S and S/A devices showed prompt decays to the half intensity, and these are clearly different from the delayed decay of the $\mathrm{D} / \mathrm{A}$ and $\mathrm{D} / \mathrm{S} / \mathrm{A}$ devices.

The donor might be in close proximity to the acceptor if the spacer layer was not deposited uniformly. Therefore, we carefully checked the surface morphology of the donor layer, the spacer on the donor layer, and the acceptor layer on the spacer/ donor stacked layer (Fig. S3†). They were sufficiently smooth with an average roughness $\left(R_{\mathrm{a}}\right)$ of $0.37 \mathrm{~nm}$ for the donor layer and $0.33 \mathrm{~nm}$ for the spacer layer. The acceptor layer on the spacer/donor stacked layer showed a relatively rough surface morphology with an $R_{\mathrm{a}}$ of $0.84 \mathrm{~nm}$ and the maximum depth of the depressions was $6 \mathrm{~nm}$ (Fig. S3c $\dagger$ ). These are much shallower than the DCA thickness of $10 \mathrm{~nm}$ and DMA thickness of $30 \mathrm{~nm}$. These results suggest that the donor TAPC and acceptor DCA are separated by the uniformly deposited spacer DMA and their distances are fairly constant in the stacked layers.

It should be noted that injected holes in the devices reach the acceptor layer, because one of the two EL emissions at the short wavelength is clearly ascribed to the emission of DCA. The onset of the DMA emission is located at an explicitly shorter wavelength than that of DCA, and the shape of the DMA emission shows three vibrational modes that differ from the unimodal shape of the DCA emission (Fig. 1b). This is also supported by the result that the hole mobility of DMA is larger than its electron mobility, as estimated by unipolar device experiments (Fig. S4†), and the charge recombination zone is located primarily in the acceptor layer. Therefore, partially accumulated holes at the interface between the donor layer and the spacer layer $(\mathrm{D} / \mathrm{S})$ interact with the electrons at the interface between the spacer layer and the acceptor layer $(\mathrm{S} / \mathrm{A})$ through the spacer layer.

It is known that TAPC sometimes shows distinctive electromer emissions in OLEDs at similar wavelength ranges to that of the exciplex emission of TAPC/DCA. ${ }^{21,22}$ However, we can rule out the possibility that the TAPC electromer is the origin of the above mentioned abnormal emissions for a number of reasons. These were confirmed by the device without both the spacer DMA and the acceptor DCA, fabricated with a structure of ITO/ PEDOT:PSS (30 nm)/TAPC (15 nm)/B3PyPB (60 nm)/LiF (1 nm)/ Al. First, the charge recombination zone is mainly located at the acceptor layer as mentioned above. Second, the maximum emission wavelength of the TAPC electromer is $581 \mathrm{~nm}$, which clearly differs from that of the exciplex emissions (Fig. S5†). Third, the transient EL decay of the TAPC electromer is very rapid unlike the exciplex emissions. The slightly delayed component originates from the exciplex emission between TAPC and B3PyPB at $432 \mathrm{~nm}$.

Considering the exciplex emission between the donor and the acceptor through the spacer layer, the type of donor molecule must influence the emission wavelength of the exciplex due to the change of the energy difference between the donor and acceptor. Four different donor molecules were used for comparison with TAPC: tris(4-carbazoyl-9-ylphenyl)amine (TCTA), $\quad N, N^{\prime}$-di(1-naphthyl)- $N, N^{\prime}$-diphenyl-(1,1'-biphenyl)-4, $4^{\prime}$ diamine (NPD), $N, N^{\prime}$-bis[4-[bis(3-methylphenyl)amino]phenyl]$N, N^{\prime}$-diphenyl-[1,1'-biphenyl]-4,4'-diamine (DNTPD), and 4,4', $4^{\prime \prime}$-tris[(3-methylphenyl)phenylamino]triphenylamine (MTDATA). Chemical structures, energy diagrams, PL spectra of the co-evaporated films with the acceptor DCA, and EL spectra 
of the devices with the same structure as the TAPC donor device are shown in Fig. 3. The PL spectra of the co-evaporated films were bathochromically shifted with decreased energy gaps between the HOMO of the donor and the LUMO of the acceptor DCA. TCTA and NPD showed emissions corresponding to their exciplex with the acceptor DCA, in addition to the emission from the acceptor DCA itself. In the devices with DNTPD and MTDATA, the emission corresponding to their exciplex with the acceptor DCA also appeared, but a blue-shifted emission corresponding to their exciplex with the spacer DMA appeared more strongly because of their shallower HOMO energy level compared to that of NPD, TCTA, and TAPC, resulting in a larger hole injection barrier to the spacer layer, efficiently forming the exciplex with the spacer DMA. These results showing that the lowest-energy band emissions are clearly dependent on the energy difference between the donor and the acceptor species demonstrate that these emissions are related to the exciplex between the donor and the acceptor species.

We performed steady-state PL measurements, to further probe the emission from the long-distance coupled exciplex, on a stacked film of TAPC (2 nm)/DMA (10 nm)/DCA (2 nm), a stacked film of TAPC ( $2 \mathrm{~nm}) / \mathrm{DCA}(2 \mathrm{~nm})$, and a mixed film of TAPC:DCA (20 nm). Excitation light (380-405 nm) was incident on the side of the DCA layer. Without any electric field, the stacked TAPC/DMA/DCA film exhibited an intense low-energy band PL with a $\lambda_{\max }$ of $534 \mathrm{~nm}$ (Fig. S6†), ruling out the possibility of an electromer (or electroplex) emission. For comparison, the stacked TAPC/DCA film and the mixed TAPC:DCA film exhibited low-energy band PL with a $\lambda_{\max }$ of 548 $\mathrm{nm}$ and $561 \mathrm{~nm}$, respectively. This observed blue shift in the PL spectra is consistent with the increased spatial separation of the coupled hole and electron in the following order: the mixed TAPC:DCA film $<$ the stacked TAPC/DCA film $<$ the stacked TAPC/DMA/DCA film, because the spatial separation leads to a decrease in the coulombic attraction energy in the holeelectron pair and thus increases the exciplex emission energy. ${ }^{14,23}$

Furthermore, the net intensity of the low-energy band emission was decreased in the presence of atmospheric oxygen (Fig. S7 $\uparrow$ ), indicating that the triplet excited state is involved in the emission mechanism, as in the case of the previously reported exciplex systems exhibiting TADF.,18 The excited state

a
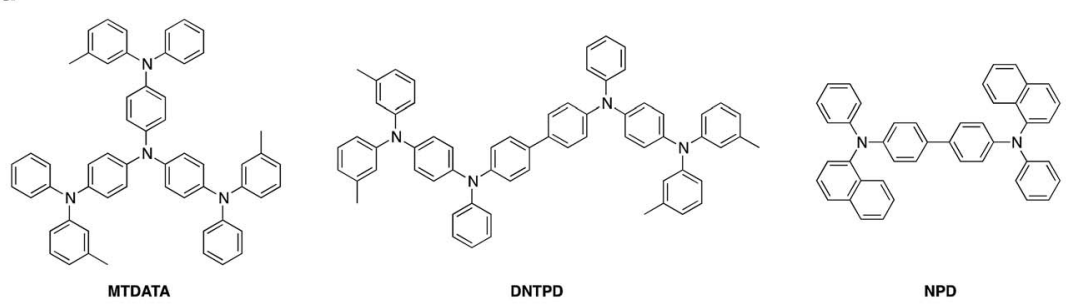

NPD

C
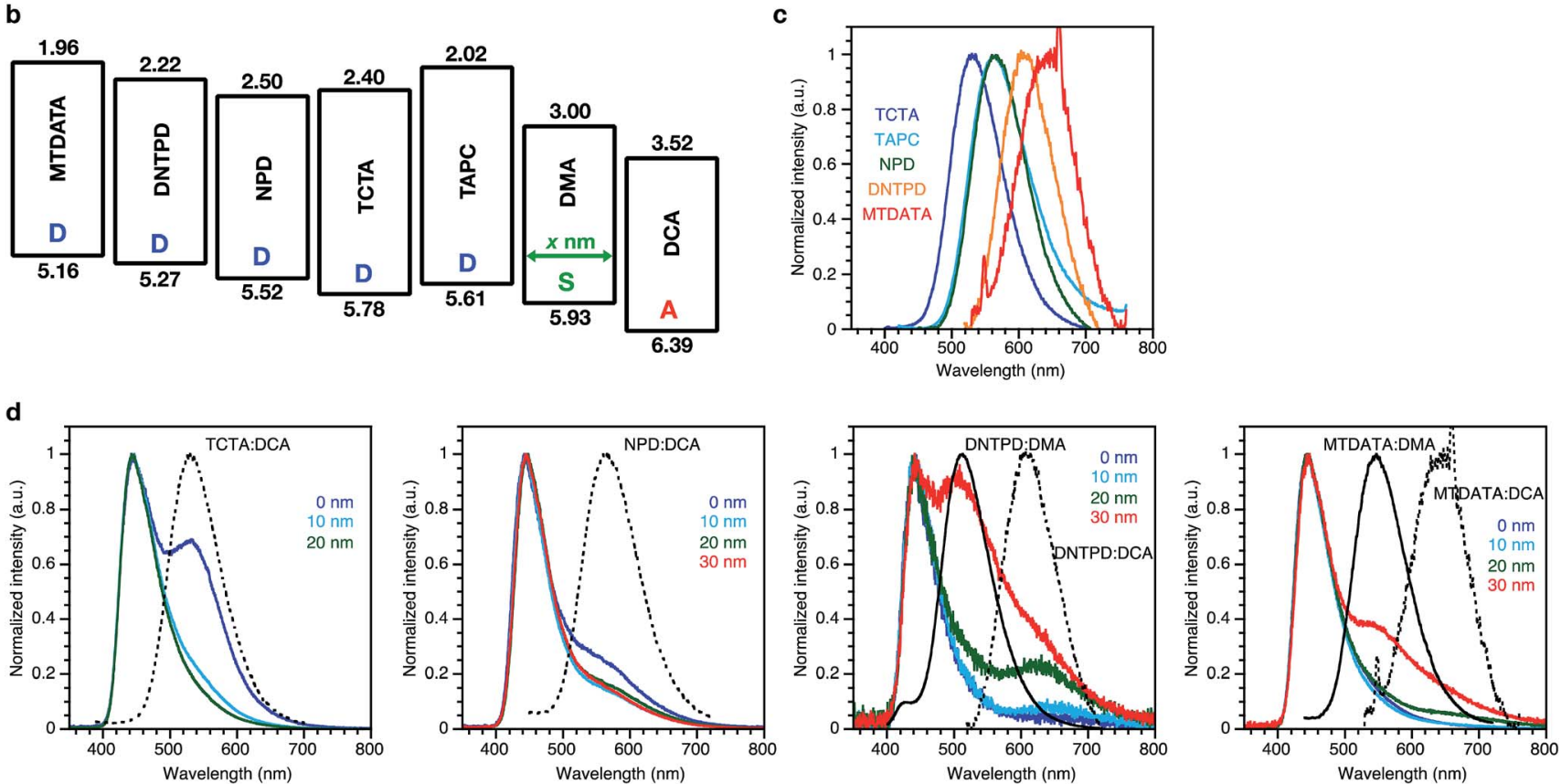

Fig. 3 EL properties of the devices with different types of donor compounds; (a) chemical structures of the compounds. (b) Energy diagrams of the compounds. (c) PL spectra of the co-evaporated (1/1 wt/wt) films of DCA and the different donors: TCTA ( $\left.\lambda_{\text {ex }} 370 \mathrm{~nm}\right)$, NPD $\left(\lambda_{\text {ex }} 380 \mathrm{~nm}\right)$, DNTPD $\left(\lambda_{\text {ex }} 380 \mathrm{~nm}\right)$, and MTDATA $\left(\lambda_{\text {ex }} 400 \mathrm{~nm}\right)$. (d) EL spectra at $25 \mathrm{~mA} \mathrm{~cm}^{-2}$ with different spacer thicknesses. The black solid and dashed lines represent the $\mathrm{PL}$ spectra of the corresponding exciplexes. 
can be quenched by energy transfer and/or electron transfer to oxygen. However, the PL intensity was recovered by removing oxygen in a vacuum, although photodegradation was still observed even under vacuum. This result suggests that the quenching process is unlikely based on an irreversible chemical reaction with oxygen and likely governed by the triplet energy transfer to oxygen. The peak intensity of the high-energy band emission at $437 \mathrm{~nm}$ corresponding to the PL of DMA itself (Fig. S7c $\dagger$ ) did not show reversible oxygen quenching, also supporting the exciplex nature of the low-energy band emission.

Transient PL decay measurements on the stacked TAPC/ DMA/DCA film and the mixed TAPC:DCA film revealed multiexponential decays with significant contribution of the submicrosecond delayed emission, along with nanosecond prompt emission (Fig. S8 and Table S5-S7†). The lifetimes of these delayed emissions decreased with increasing the temperature, suggesting thermal activation of the triplet excited state back to the singlet excited state through reverse intersystem crossing (RISC). The rate constants of RISC follow Arrhenius-type temperature dependence with an activation energy of $10 \mathrm{meV}$ for the mixed TAPC:DCA film and $4 \mathrm{meV}$ for the stacked TAPC/ DMA/DCA film (Fig. S9 $\dagger$ ). The decreased activation energy of RISC is attributed to the increased spatial separation of the coupled hole and electron in the stacked film, having a very small electron exchange energy (i.e., energy difference between the singlet and triplet excited states). ${ }^{\mathbf{2 4 2 5}}$ We note that the exciplex emission intensity showed a linear dependence on the excitation power (Fig. S10 $\dagger$ ), confirming that the emission is caused through a unimolecular process, rather than a bimolecular process, such as triplet-triplet annihilation.

The Onsager radius for typical organic materials is known to be $16-19 \mathrm{~nm}$ with a dielectric constant of 3.0-3.5 at room temperature. ${ }^{26}$ It is unlikely that electrons and holes can be coupled over a distance of $70 \mathrm{~nm}$ without any spacer molecules to form the exciplex state. Therefore, the role of the spacer molecules would be a key factor in understanding the mechanism of the abnormally long-distance exciplex formation; we could propose a conceivable mechanism to explain this behaviour. As an initial step, two exciplex states at the D/S and S/A interfaces, $\left[\mathrm{D}^{\delta+} \cdot \mathrm{S}^{\delta-}\right]$ and $\left[\mathrm{S}^{\delta+} \cdot \mathrm{A}^{\delta-}\right]$, are formed, respectively. Then, the negative and positive charges on the spacer species, $\mathrm{S}^{\delta-}$ and $\mathrm{S}^{\delta+}$, diffuse in the spacer layer, as expressed in (i) and (ii), towards the cathode and anode, respectively. This could be supported by the recent report that bound electronhole pairs move to a distance of $10 \mathrm{~nm}$ at the $\mathrm{p}-\mathrm{n}$ interface. ${ }^{19}$ The diffusion in this study is in the opposite direction of the applied bias. These long-distance excited CT states, $\left[\mathrm{D}^{\delta+} \cdot \mathrm{S}_{n^{-}}\right.$ $\left.\cdot \mathrm{S}^{\delta-}\right]$ and $\left[\mathrm{S}^{\delta+} \cdot \mathrm{S}_{m} \cdot \mathrm{A}^{\delta-}\right]$, encounter each other, and a further long-distance excited CT state $\left[\mathrm{D}^{\delta+} \cdot \mathrm{S}_{n+m} \cdot \mathrm{A}^{\delta-}\right]$ is formed as expressed in (iii).

$$
\begin{gathered}
{\left[\mathrm{D}^{\delta+} \cdot \mathrm{S}^{\delta-}\right] \rightarrow\left[\mathrm{D}^{\delta+} \cdot \mathrm{S} \cdot \mathrm{S} \cdot \mathrm{S}^{\delta-}\right] \rightarrow\left[\mathrm{D}^{\delta+} \cdot \mathrm{S}_{n} \cdot \mathrm{S}^{\delta-}\right]} \\
{\left[\mathrm{S}^{\delta+} \cdot \mathrm{A}^{\delta-}\right] \rightarrow\left[\mathrm{S}^{\delta+} \cdot \mathrm{S} \cdot \mathrm{S} \cdot \mathrm{A}^{\delta-}\right] \rightarrow\left[\mathrm{S}^{\delta+} \cdot \mathrm{S}_{m} \cdot \mathrm{A}^{\delta-}\right]} \\
{\left[\mathrm{D}^{\delta+} \cdot \mathrm{S}_{n} \cdot \mathrm{S}^{\delta-}\right]+\left[\mathrm{S}^{\delta+} \cdot \mathrm{S}_{m} \cdot \mathrm{A}^{\delta-}\right] \rightarrow\left[\mathrm{D}^{\delta+} \cdot \mathrm{S}_{n+m} \cdot \mathrm{A}^{\delta-}\right]}
\end{gathered}
$$

In the proposed emission mechanism, the exciplex formation at both the $\mathrm{D} / \mathrm{S}$ and S/A interfaces is important to form long-distance electron-hole coupling. We used 4,4'-bis $(N$-carbazolyl)-1,1'-biphenyl (CBP) as a spacer instead of the DMA spacer. The chemical structure, PL spectra, EL spectra and transient decay of CBP are shown in Fig. S11. $\dagger$ The co-evaporated film of TAPC:CBP (D:S) showed bathochromically shifted PL spectra compared to each neat film of TAPC and CBP, while the co-evaporated film of CBP:DCA (S:A) showed the same PL spectra as that of DCA itself. However, the PL lifetime of the CBP:DCA (S:A) film at $550 \mathrm{~nm}$ was relatively long compared to the TAPC:CBP (D:S) film, although the PL lifetime at $450 \mathrm{~nm}$, which is the wavelength at the peak top in the PL spectrum of the CBP:DCA (S:A) film, showed much faster decay. The decays of the neat film of CBP and DCA themselves are also fast. Therefore, the delayed lifetime of the CBP:DCA mixed film at $550 \mathrm{~nm}$ does not result from the excimer of CBP or DCA, but is considered to be ascribed to the less generated exciplex species between CBP and DCA, although the PL of the CBP:DCA film was the same as that of the DCA neat film without any other lowenergy band emission. The D/S/A-type OLEDs with the CBP spacer also showed two EL emissions. One with a $\lambda_{\max }$ of 445 $\mathrm{nm}$ is ascribed to emissions from the acceptor DCA itself, while the other at $\lambda_{\max }=550 \mathrm{~nm}$ is ascribed to the exciplex emission between the donor TAPC and the acceptor DCA. The device with spacer distances greater than $15 \mathrm{~nm}$ did not show the exciplex EL. This spacer thickness is still exceptionally large, but is smaller than that of the device with the DMA spacer. This result is attributed to the less exciplex formation at the S/A interface.

We investigated the effect of spacer thickness on the exciplex species generated by photoexcitation (Fig. S12 and Table S8S9 $\dagger$ ). Stacked films were prepared as quartz/TAPC (2 nm)/DMA or CBP $(x \mathrm{~nm}) / \mathrm{DCA}(2 \mathrm{~nm})$. Excitation light was irradiated on the acceptor DCA side at 380-405 $\mathrm{nm}$. When DMA was used as a spacer, incident photons were absorbed in the DCA layer and unabsorbed photons passing through the DCA layer were also absorbed in the DMA layer. This is supported by the PL spectra showing DMA emissions at shorter wavelengths than those of DCA. Therefore, the exciplex states at both the D/S and S/A interfaces can be generated initially, and then the D/A exciplex forms as well as the electrical excitation. Up to a $70 \mathrm{~nm}$ DMA spacer layer, an emission corresponding to the D/A exciplex at 530-550 $\mathrm{nm}$ appeared. This thickness is larger than the 5-20 $\mathrm{nm}$ CBP spacer in which the D/A exciplex emission was observed. As shown in Fig. S11b, $\dagger$ the S/A exciplex state is less generated. Therefore, for the formation of the D/A exciplex, $\mathrm{S}^{\delta-}$ needs to move to the acceptor DCA layer, which is why the CBP spacer thickness was smaller than that of the DMA spacer in the formation of the long-distance D/S/A exciplexes.

Finally, the current density and voltage dependence of the D/ A exciplex formation as shown in Fig. S13 $\uparrow$ also supports this proposed mechanism. Under a positive bias, the negative and positive spacer species $\mathrm{S}^{\delta-}$ and $\mathrm{S}^{\delta+}$ become less diffusive toward the cathode and anode, respectively, as the electric field increases. Therefore, it becomes difficult to form long-distance coupled $\left[\mathrm{D}^{\delta+} \cdot \mathrm{S}_{n+m} \cdot \mathrm{A}^{\delta-}\right]$, in which $n$ and $m$ become small, and 
consequently the emission corresponding to the D/A exciplex with the spacer decreases.

\section{Conclusion}

Exciplex emissions were observed with exceptionally longdistance donor-acceptor coupling through spacer molecules in OLEDs, with a maximum spacer distance of $70 \mathrm{~nm}$. The observed low-energy band EL component was congruent with the exciplex PL in the co-evaporated films of the donor and acceptor molecules, even when the donor and acceptor were separated by a long-distance spacer up to around $70 \mathrm{~nm}$. Four different types of donor molecules having different HOMO levels also showed different low-energy band EL components with the acceptor DCA, corresponding to the difference of their HOMO levels and LUMO levels of the acceptor DCA. The delayed transient decay and large oxygen dependency of the low-energy band emission are considered to originate from the feature of the exciplex species. The effect of the different spacer molecules suggests that the exciplex formation both at the donor/spacer and the spacer/acceptor interface results in long-distance coupling between the donor and the acceptor through such a thick spacer layer. This finding of the exceptionally longdistance donor-acceptor coupling in OLEDs is far beyond previous perception and helps to eliminate preconceptions of photophysics in organic semiconductors, and would have the possibility to lead to totally different highly sensitive sensors or photodetectors to external stimulus, with various applications other than OLED applications.

\section{Conflicts of interest}

There are no conflicts to declare.

\section{Acknowledgements}

Y.-J. Pu gratefully acknowledges PRESTO (13417316) from JST, and a Grant-in-Aid for Young Scientists [A] (24685029) and a Grant-in-Aid for Scientific Research [B] (17H03103) from the JSPS.

\section{References}

1 M. R. Wasielewski, Chem. Rev., 1992, 92, 435-461.

2 D. Gust, T. A. Moore and A. L. Moore, Acc. Chem. Res., 2001, 34, 40-48.

3 S. Fukuzumi, Phys. Chem. Chem. Phys., 2008, 10, 2283-2297.

4 N. Matsumoto and C. Adachi, J. Phys. Chem. C, 2010, 114, 4652-4658.

5 E. Skuodis, A. Tomkeviciene, R. Reghu, L. Peciulyte, K. Ivaniuk, D. Volyniuk, O. Bezvikonnyi, G. Bagdziunas, D. Gudeika and J. V. Grazulevicius, Dyes Pigm., 2017, 139, 795-807.
6 K. Goushi, K. Yoshida, K. Sato and C. Adachi, Nat. Photonics, 2012, 6, 253-258.

7 Y. S. Park, S. Lee, K. H. Kim, S. Y. Kim, J. H. Lee and J. J. Kim, Adv. Funct. Mater., 2013, 23, 4914-4920.

8 J. W. Sun, J. H. Lee, C. K. Moon, K. H. Kim, H. Shin and J. J. Kim, Adv. Mater., 2014, 26, 5684-5688.

9 D. Veldman, S. C. J. Meskers and R. A. J. Janssen, Adv. Funct. Mater., 2009, 19, 1939-1948.

10 C. Deibel, T. Strobel and V. Dyakonov, Adv. Mater., 2010, 22, 4097-4111.

11 J. Baek, T. Umeyama, K. Stranius, H. Yamada, N. V. Tkachenko and H. Imahori, J. Phys. Chem. C, 2017, 121, 13952-13961.

12 T. Umeyama, T. Hanaoka, J. Baek, T. Higashino, F. AbouChahine, N. V. Tkachenko and H. Imahori, J. Phys. Chem. C, 2016, 120, 28337-28344.

13 J. R. Miller, L. T. Calcaterra and G. L. Closs, J. Am. Chem. Soc., 1984, 106, 3047-3049.

14 H. Nakanotani, T. Furukawa, K. Morimoto and C. Adachi, Sci. Adv., 2016, 2, e1501470.

15 M. Chapran, E. Angioni, N. J. Findlay, B. Breig, V. Cherpak, P. Stakhira, T. Tuttle, D. Volyniuk, J. V. Grazulevicius, Y. A. Nastishin, O. D. Lavrentovich and P. J. Skabara, ACS Appl. Mater. Interfaces, 2017, 9, 4750-4757.

16 B. B. Li, L. Gan, X. Y. Cai, X. L. Li, Z. H. Wang, K. Gao, D. C. Chen, Y. Cao and S. J. Su, Adv. Mater. Interfaces, 2018, 5, 1800025.

17 G. Sych, J. Simokaitiene, O. Bezvikonnyi, U. Tsiko, D. Volyniuk, D. Gudeika and J. V. Grazulevicius, J. Phys. Chem. C, 2018, 122, 14827-14837.

18 M. Colella, A. Danos and A. P. Monkman, J. Phys. Chem. Lett., 2019, 10, 793-798.

19 P. B. Deotare, W. Chang, E. Hontz, D. N. Congreve, L. Shi, P. D. Reusswig, B. Modtland, M. E. Bahlke, C. K. Lee, A. P. Willard, V. Bulovic, T. Van Voorhis and M. A. Baldo, Nat. Mater., 2015, 14, 1130-1134.

20 H. Sasabe, E. Gonmori, T. Chiba, Y. J. Li, D. Tanaka, S. J. Su, T. Takeda, Y. J. Pu, K. I. Nakayama and J. Kido, Chem. Mater., 2008, 20, 5951-5953.

21 S. Y. Yang and M. X. Jiang, Chem. Phys. Lett., 2009, 484, 5458.

22 J. Kalinowski, G. Giro, M. Cocchi, V. Fattori and P. Di Marco, Appl. Phys. Lett., 2000, 76, 2352-2354.

23 M. Cocchi, D. Virgili, C. Sabatini and J. Kalinowski, Chem. Phys. Lett., 2006, 421, 351-355.

24 A. Kohler and D. Beljonne, Adv. Funct. Mater., 2004, 14, 1118.

25 A. Endo, K. Sato, K. Yoshimura, T. Kai, A. Kawada, H. Miyazaki and C. Adachi, Appl. Phys. Lett., 2011, 98, 83302. 26 L. Onsager, Phys. Rev., 1938, 54, 554-557. 